\title{
Characterization of a glucose tolerant $\beta$-glucosidase from Aspergillus unguis with high potential as a blend-in for biomass hydrolyzing enzyme cocktails
}

\author{
Prajeesh Kooloth Valappil • Kuni Parambil Rajasree • Amith Abraham • \\ Meera Christopher $\cdot$ Rajeev K. Sukumaran (i)
}

Received: 9 May 2019/Accepted: 17 August 2019/Published online: 5 September 2019

(C) Springer Nature B.V. 2019

\begin{abstract}
Objectives Characterization of glucose tolerant beta glucosidase (GT-BGL) secreted by Aspergillus unguis NII 08123, determination of the gene and protein sequences of the enzyme and establishing its performance in blends for lignocellulose hydrolysis.

Results Supplementation of A. unguis beta glucosidase (BGL) to cellulase released 1.6 times more sugar within $12 \mathrm{~h}$ during the hydrolysis of lignocellulosic biomass. The enzyme was determined to be similar to BGL-F from Emericella nidulans by MALDI-TOF analysis, and was found to be a GH3 family protein. Molecular Docking simulation studies showed that the enzyme has lesser affinity for glucose $(-5.7 \mathrm{kcal} /-$ mol) compared to its substrate cellobiose $(-7.5 \mathrm{kcal} / \mathrm{mol})$. The residues present in the $N$ -
\end{abstract}

Electronic supplementary material The online version of this article (https://doi.org/10.1007/s10529-019-02724-z) contains supplementary material, which is available to authorized users.

P. Kooloth Valappil · K. P. Rajasree · A. Abraham ·

M. Christopher · R. K. Sukumaran $(\square)$

Biofuels and Biorefineries Section, Microbial Processes and Technology Division, CSIR-National Institute for Interdisciplinary Science and Technology, Industrial

Estate P.O, Trivandrum 695019, India

e-mail: rajeevs@niist.res.in

P. Kooloth Valappil · M. Christopher

Ghaziabad, India terminal domain are mostly involved in bond formation with both the substrate and the product, while the $C$-terminal domain contains the catalytic region. Insilico studies showed that its predicted structure is unlike that of previously reported BGLs, which might provide a clue to its exceptional catalytic activity.

Conclusion The GT-BGL from A. unguis NII 08123 was proven effective as a blend in for biomass hydrolyzing enzyme cocktails and the possible reasons for its glucose tolerance was determined through studies on its modeled structure.

Keywords $\beta$-glucosidase - Glucose tolerant . Aspergillus unguis · Biomass hydrolysis - Genome · Homology model

\author{
A. Abraham \\ Department of Chemical Engineering, Hanyang \\ University, Seoul, Republic of Korea \\ Present Address: \\ K. P. Rajasree \\ Department of Microbiology and Cell Biology, Indian Institute \\ of Science, Bangalore 560012, India
}




\section{Introduction}

Conversion of the renewable lignocellulosic biomass to valuable products, including fuels, chemicals and chemical building blocks, is of strategic importance for sustainable development and energy security ( $\mathrm{Wu}$ et al. 2016). Plant biomass is composed primarily of cellulose (35-50\%), hemicellulose $(20-35 \%)$ and lignin (15-30\%), and its degradation is mediated by the hydrolytic enzymes cellulases and hemicellulases (Tabanag and Tsai 2018). Importantly, there are three classes of enzymes involved in cellulose breakdown(1) endoglucanases which produce nicks in the cellulose polymer, thereby exposing reducing and non-reducing ends (2) exoglucanases, including d-cellodextrinases and cellobiohydrolases, which act on these reducing and non-reducing ends to liberate cellobiose units and (3) $\beta$-glucosidase (BGL) which cleave the cellobiose to liberate glucose. Majority of the microbes do not have a complete cellulolytic system capable of generating glucose from natural lignocellulosic material (Singh et al. 2017). On the other hand, fungi are known to secrete copious amounts of cellulases. The cellulolytic systems of most fungi are inducible and are tightly regulated by catabolite repression, which means that the organism produces cellulases only when cellulose or certain disaccharide hydrolysis products or their derivatives (e.g., sophorose, gentiobiose, cellobiose) are present in the medium, and the expression of cellulase genes is shut down in the presence of easily utilizable sugars like glucose (Amore et al. 2013). In several fungi, BGL is produced in lower quantities compared to the other two classes of enzymes, and it is slow acting, making BGL the rate limiting component in the hydrolysis of cellulose (Saloheimo et al. 2002). The BGL is also subject to inhibition by glucose and cellobiose.

This whole mechanism of induction and repression thus helps the fungus to save energy by eliminating the need to synthesize cellulases continuously when they are not required. However, this economy practiced by the fungus is not desirable when they are to be used as an industrial source of cellulases. From the foregoing discussions, the critical role of BGL in hydrolysis becomes apparent. As one of the major limitations in the saccharification of biomass is the product inhibition of BGL by glucose; and this key enzyme has to be present in sufficient quantities in the enzyme cocktail used for biomass hydrolysis, for it to be efficient. Also, it is desirable for the enzyme to have better specific activity and to be tolerant to glucose.

The major sources of $\beta$-glucosidases are microorganisms like bacteria, filamentous fungus and yeast. Among these, filamentous fungi are considered as the most potent $\beta$-glucosidase producers (Tiwari et al. 2013). Preliminary studies in our lab showed that a fungal strain isolated from soil and identified as Aspergillus unguis produced a $\beta$-glucosidase with $K_{i}$ of $0.8 \mathrm{M}$ against glucose, which is the second highest glucose tolerance reported in the Aspergillus nidulans group (Rajasree et al. 2013). Among the several isoforms of BGL secreted by this fungus, a low molecular weight protein was identified as the highly glucose tolerant $\beta$-glucosidase (GT-BGL). This enzyme was produced in small quantities in response to induction by lignocellulosic substrates or known inducers like lactose.

In order to fully exploit the exceptional properties of this enzyme, it is necessary to understand the protein structure and sequence. The current study analyzed both genome-level and protein-specific data for elucidating the sequence of the A. unguis GT-BGL, and to use this, for gaining information on its structure and the molecular mechanisms behind its glucose tolerance. The performance of this enzyme in blends with different cellulases has also been evaluated. This information is expected to help understanding the regulation of BGL expression in this fungus, which in turn is needed for enhancing the production of this enzyme in commercially viable scales.

\section{Materials and methods}

\section{Organism}

Cultures of A. unguis NII 08123 were maintained on Potato Dextrose Agar (PDA) at $30{ }^{\circ} \mathrm{C}$. Five days old sporulated PDA slants were used for enzyme production. The organism was sub-cultured at regular intervals and stored at $4{ }^{\circ} \mathrm{C}$.

Production of $\beta$-glucosidase and zymogram and MALDI-TOF analyses for determination of the GT-BGL

$\beta$-Glucosidase from A. unguis was produced and assayed as described previously (Rajasree et al. 2013). 
For zymogram analysis, $12 \%$ acrylamide gels without denaturing agents were used. Protein concentration was estimated by Bradford assay (Bradford 1976) and normalized before sample loading. For comparative analysis, two gels were cast, and both gels were loaded with replicates of the same samples. After electrophoresis, the gels were washed with distilled water and incubated in a $10 \mathrm{mM}$ solution of 4-Methylumbelliferyl $\beta$-D-glucopyranoside (MUG) in citrate buffer $(0.05 \mathrm{M}, \mathrm{pH} 4.8)$ for $10 \mathrm{~min}$ at $50{ }^{\circ} \mathrm{C}$. Glucose tolerant proteins were detected by incubating one of the gels in a MUG solution containing 0.5 M glucose. The protein bands were visualized under UV. After separation by polyacrylamide gel electrophoresis and visualization using MUG, the band corresponding to BGL3 was excised from the gel and electro-eluted into sodium citrate buffer $(0.05 \mathrm{M}, \mathrm{pH} 4.8)$. The protein was then digested with trypsin according to the protocol of (Trauger et al. 2002). The MS/MS spectra were acquired in the positive ion mode using a AximaCFR Plus MALDI-TOF Mass Spectrometer from Shimadzu, Japan. Database search for protein identification was performed using the MASCOT search engine. The database query used was for microbial species against the SWISS-PROT and NCBI nonredundant databases, using only mono isotopic masses. MS-BLAST search was performed for sequence homology-based protein identity.

\section{Cellobiase activity of A. unguis GT-BGL}

Xylan-grown A. unguis cultures were filtered to remove the biomass. The filtrate was concentrated and then separated by $12 \%$ polyacrylamide gel electrophoresis. The gels were stained using MUG to visualize BGLs, and the band corresponding to GTBGL was excised. The protein was electro-eluted from the gel and concentrated by lyophilization. The protein concentrate was then assayed for cellobiase activity by the protocol of Ghose (1987). Briefly, the protein was incubated for $30 \mathrm{~min}$ at $50{ }^{\circ} \mathrm{C}$ with $15 \mathrm{mM}$ cellobiose, after which the mixture was analyzed by HPLC for the release of glucose. Appropriate substrate and enzyme blanks were included.

Hydrolysis of lignocellulosic biomass

Alkali pretreated rice straw (PT-RS) produced as per previously optimized protocols (Christopher et al.
2017) was used for analyzing the saccharification efficiency of enzyme blends containing A. unguis BGL. Cellulases from Penicillium janthinellum and BGL from A. unguis was produced according to protocols optimized in the laboratory and was assayed as per IUPAC methods (Ghose 1987). Hydrolysis was carried out in $150 \mathrm{ml}$ screw capped conical flasks with a working volume of $30 \mathrm{ml}$. The biomass loading was $10 \% \mathrm{w} / \mathrm{w}$, the cellulase loading was $20 \mathrm{FPUs} / \mathrm{g}(0.606$ $\mathrm{ml})$ and BGL loading was $60 \mathrm{IU} / \mathrm{g}(3.75 \mathrm{ml})$. The reactants were incubated at $50{ }^{\circ} \mathrm{C}$ for $48 \mathrm{~h}$, with sampling at every $12 \mathrm{~h}$. Controls without BGL supplementation and with supplementation of $60 \mathrm{IU} / \mathrm{g}$ of commercial BGL preparations were run in parallel. Sugar release was monitored as total reducing sugar by the DNS method (Miller 1959).

Selection of the best inducer of BGL in A. unguis

A number of easily available carbon sources were checked for inducing the production of extracellular proteins and BGL by A. unguis NII 08123. The organism was grown in Mandels and Weber (MW) Medium (Mandels and Weber 1969) containing 1.0\% glucose-as carbon source for $48 \mathrm{~h}$ to build up the biomass. The fungal biomass was then separated by filtration, washed with distilled $\mathrm{H}_{2} \mathrm{O}$ to remove residual glucose, and then transferred to fresh MW medium (Mandels and Weber 1969) containing 1.0\% $(\mathrm{w} / \mathrm{v})$ of one of the inducers-Glucose, Xylan, Cellulose, Cellobiose, Lactose, Wheat bran, Rice straw, Alkali-pretreated Rice Straw (PT-RS), Maltose or Fructose). Cultures were incubated at $30 \pm 2{ }^{\circ} \mathrm{C}$ and $200 \mathrm{rpm}$ for $120 \mathrm{~h}$. Samples were collected at $24 \mathrm{~h}$ intervals for BGL assay and zymogram analysis.

In-silico characterization of BGL

The sequences encoding BGLs in A. unguis NII 08123 were obtained from the whole genome sequence data (generated through whole genome shotgun sequencing and submitted to GenBank with ID QLOF01000000 [unpublished results]) by using BLAST analysis to identify motifs conserved in $\beta$-glucosidases. The amino acid sequences of the proteins were obtained by translation using the EXPASY tool (Gasteiger et al. 2003). 


\section{Physicochemical characterization}

Intrinsic properties like molecular weight, isoelectric point (pI), half-life, aliphatic index, instability index and Grand Average Hydropathy (GRAVY) were predicted using the EXPASY tool ProtParam (Gasteiger et al. 2003). The presence of signal peptides was checked using the SignalP program (Petersen et al. 2011).

\section{Homology modelling of BGL structure}

The three-dimensional structures of the proteins were homology modeled using the SWISS-MODEL workspace (Biasini et al. 2014), using appropriate templates. GT-BGL was modeled using the Aspergillus oryzae Family GH3 beta-D glucosidase structure (PDB ID-5FJJ) as template (Agirre et al. 2016). Visualization and analysis of the models were done using the Swiss PDB Viewer (Guex and Peitsch 1997) and PyMOL programs (DeLano 2002).

\section{Docking studies}

Computer-aided molecular docking of the substrates to the active site of modeled structures of the proteins was done using AutoDock (Goodsell and Olson 1990). Initial coordinates of the substrates (Cellobiose and Glucose) were obtained from the PDB database. The modeled structure of the protein was taken as the receptor protein. The binding energy of each complex was calculated and analyzed.
Screening and amplification of GT-BGL

from the genome of A. unguis

The putative sequence of the highly glucose tolerant BGL (GT-BGL) of A. unguis was obtained from MALDI-MS analysis of the partially purified protein. The sequences of the peptide fragments were compared with those of the predicted secretory BGLs in order to identify the GT-BGL. PCR primers BGLF_F1 (TAGGTTCCGCCCTGAGGATCTTGG) and BGLF_R1 (CGTTTACGGGCTCACCGTCTTTC) were designed for amplification of the full coding sequence, and primer pairs BGLCAT_F1 (GCCAGAGATGCAATCACG) and BGLCAT_R1 (CCGCGTGATATCCATGGT) were used for amplification of the catalytic domain of the GT-BGL. The amplicons were then sequenced using standard protocols at the CSIR-NIIST DNA sequencing facility for confirmation.

\section{Results}

Hydrolysis of alkali pretreated rice straw using enzyme blends

Hydrolysis of the alkali pretreated biomass using $P$. janthinellum cellulase (at $20 \mathrm{FPU} / \mathrm{g}$ biomass enzyme loading) yielded $247 \mathrm{mg} / \mathrm{g}$ reducing sugar (25\% of theoretical maximum efficiency) in $12 \mathrm{~h}$. When supplemented with $60 \mathrm{IU}$ of A. unguis BGL per gram biomass, a reducing sugar yield of $555 \mathrm{mg} / \mathrm{g}$ (55\% of theoretical maximum) was obtained within $12 \mathrm{~h}$ (Table 1). Maximum hydrolytic efficiency ( $85 \%$ of theoretical maximum $/ 858 \mathrm{mg} / \mathrm{g}$ ) was attained in $36 \mathrm{~h}$.

Table 1 Hydrolysis of alkali pretreated rice straw using $P$. janthinellum cellulase supplemented with A. unguis $\beta$-glucosidase or commercial BGL

\begin{tabular}{|c|c|c|c|c|c|c|}
\hline \multirow[t]{3}{*}{ Time (h) } & \multicolumn{2}{|l|}{ A. unguis } & \multicolumn{2}{|c|}{ Commercial BGL } & \multicolumn{2}{|c|}{ Control—no additional BGL } \\
\hline & \multicolumn{2}{|l|}{$60 \mathrm{IU} / \mathrm{g}$} & \multicolumn{2}{|l|}{$60 \mathrm{IU} / \mathrm{g}$} & \multirow[b]{2}{*}{$\begin{array}{l}\text { Reducing } \\
\text { sugar }(\mathrm{mg} / \mathrm{g})\end{array}$} & \multirow[b]{2}{*}{$\begin{array}{l}\text { Efficiency } \\
(\%)\end{array}$} \\
\hline & $\begin{array}{l}\text { Reducing } \\
\text { sugar }(\mathrm{mg} / \mathrm{g})\end{array}$ & $\begin{array}{l}\text { Efficiency } \\
(\%)\end{array}$ & $\begin{array}{l}\text { Reducing } \\
\text { sugar }(\mathrm{mg} / \mathrm{g})\end{array}$ & $\begin{array}{l}\text { Efficiency } \\
(\%)\end{array}$ & & \\
\hline 12 & $555 \pm 1.9$ & 55 & $351 \pm 9.9$ & 35 & $247 \pm 12.7$ & 25 \\
\hline 24 & $834 \pm 14.7$ & 83 & $787 \pm 14$ & 78 & $696 \pm 24.8$ & 69 \\
\hline 36 & $858 \pm 15.6$ & 85 & $701 \pm 15.5$ & 70 & $738 \pm 18.4$ & 73 \\
\hline 48 & $819 \pm 21.1$ & 81 & $583 \pm 33.9$ & 58 & $708 \pm 7.4$ & 70 \\
\hline
\end{tabular}


Fig. 1 Secreted BGL activity of $A$. unguis cultures grown with different inducers

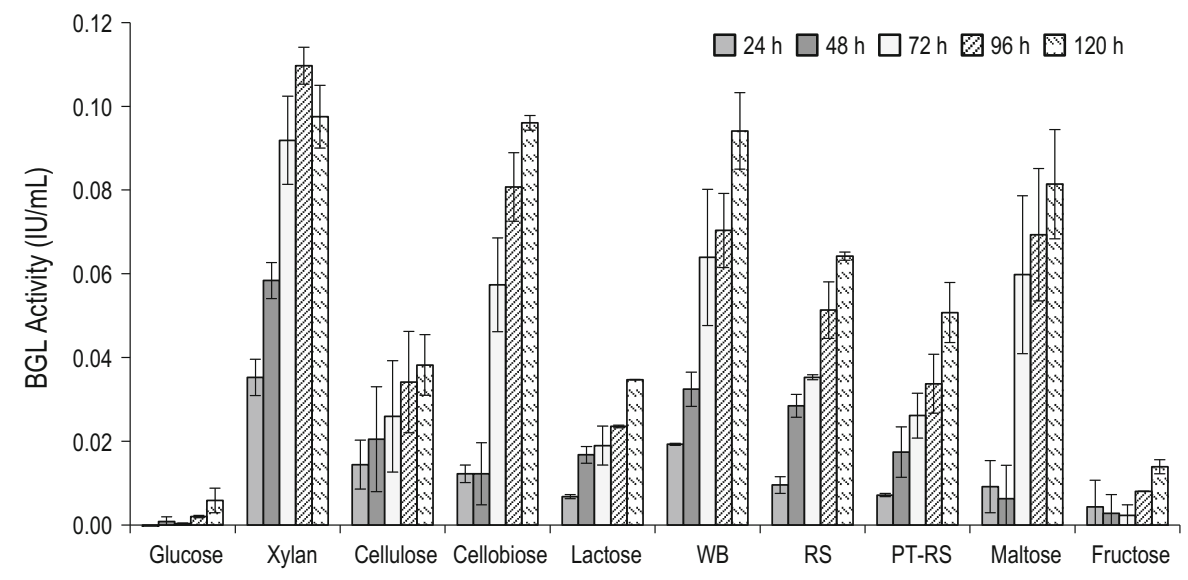

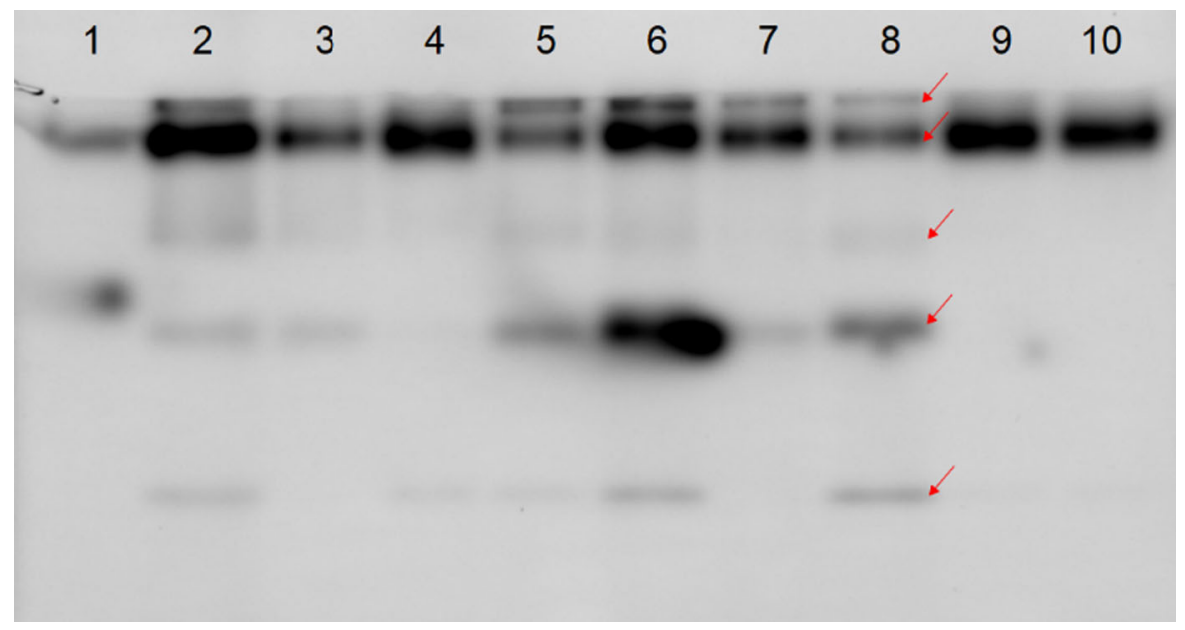

Fig. 2 Zymogram analysis of secreted BGL isoforms from $A$. unguis grown with different inducers. Lane Information: 1-glucose, 2-xylan, 3-cellulose, 4-cellobiose, 5-lactose, 6-wheat bran, 7-rice straw, 8-pretreated rice straw (PTRS), 9- maltose, 10 -fructose. Arrows indicate the position of the BGL isoforms. *Image inverted for clarity
Sugar yields for experiments performed with commercial BGL supplementation to the cellulase, was lesser than the cellulase plus A. unguis BGL combination, at all the hydrolysis durations tried. This indicates the synergy between specific cellulases and BGLs, which is a key requirement for efficient biomass hydrolyzing cocktails. While the commercial BGL used here is one of the best currently available in the market, GT-BGL from A. unguis in blends with $P$. janthinellum cellulase, could perform better than this. Apparently, BGLs from A. unguis may have better synergy with $P$. janthinellum cellulases than commercial BGLs, resulting in better hydrolytic performance.

Cellobiase activity of GT-BGL

By HPLC analysis of pure sugars, the retention times of glucose and cellobiose were determined to be 16.229 and $13.952 \mathrm{~min}$, respectively. The chromatogram of the test sample showed a peak at 16.116 min indicating presence of glucose. No such peak was detected in the control samples, thereby 


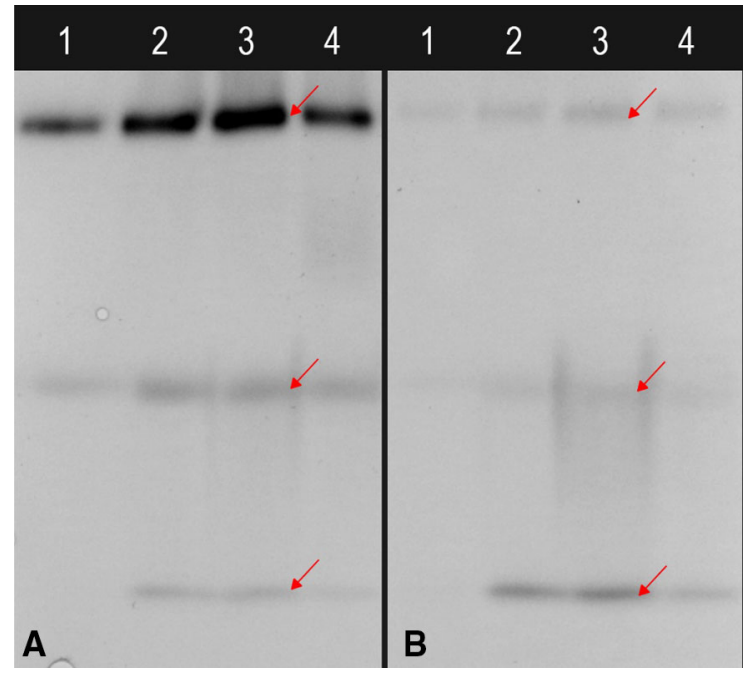

Fig. 3 Zymogram analysis of secreted BGL isoforms from xylan grown $A$. unguis in presence and absence of glucose. Zymogram performed in the absence (a) and presence (b) of $0.5 \mathrm{M}$ glucose on $A$. unguis culture supernatants from 24, 48, 72 and $96 \mathrm{~h}$ xylan grown cultures (Lanes 1, 2, 3 and 4 respectively in both $\mathbf{a}$ and $\mathbf{b})$. Arrows indicate the position of the BGL isoforms. *Image inverted for clarity

confirming that the glucose is released from cellobiose due to enzyme action, confirming that the enzyme is indeed a beta glucosidase.

\section{Partial purification and identification of glucose tolerant $\beta$-glucosidase by zymogram and MALDI- TOF analysis}

On comparing A. unguis cultures grown in media containing different inducers, the maximum BGL activity of $0.11 \mathrm{IU} / \mathrm{ml}$ was observed in the xylan induced culture (Fig. 1). The zymogram revealed that the organism secretes multiple $\beta$-glucosidases (Fig. 2) and five isoforms of the BGL were detected when the fungus was grown in xylan, lactose, wheat bran or pretreated rice straw as carbon source. BGLs from xylan induced cultures were tested for activity in presence of $0.5 \mathrm{M}$ glucose in a separate zymogram, which showed the predominant presence of three major bands which included the proposed GT BGL. Proteins corresponding to these activity bands were named as BGL1, BGL2 and BGL3 (Fig. 3). BGL1 is the high molecular weight isoform and with the highest expression among the three. On the other hand, BGL3 had the lowest molecular weight among these isoforms and it was identified as the GT-BGL as it persisted even on incubation with $0.5 \mathrm{M}$ glucose (Fig. 3).

Based on its relative position on $12 \%$ Native poly acrylamide gel electrophoresis (Native PAGE), BGL3 was speculated to be a low molecular weight protein (Rajasree et al. 2013). MALDI-TOF-MS analyses revealed the presence of two peptide fragments, showing identity with the glycosyl hydrolase $C$ terminal domain of Emericella nidulans $\beta$-glucosidase F (Table 2 and Supplementary Fig. S1), a region common to all glycosyl hydrolase family 3 (GH3) members. The GH3 $C$-terminal domain (pfam00933) is involved in the catalysis and may also be involved in the binding of $\beta$-glucan.

Identification of putative glucose-tolerant $\beta$ glucosidase (GT-BGL) from the A. unguis genome

Whole genome shot gun sequencing of the A. unguis was performed using Next Generation Sequencing and the genome sequence was submitted to Genbank with Accession Number QLOF01000000 (unpublished results). Glycoside hydrolase $(\mathrm{GH})$ family proteins were identified using the Multiple EM for Motif Elicitation (MEME) server (Bailey et al. 2006) from among the predicted proteins obtained from genome data. Motif families were identified by sequence

Table 2 Confirmation of GH3 family peptide matches from the BGL3 protein through MALDI-TOF-MS analysis

\begin{tabular}{lcccccl}
\hline Peak name & Start & End & Observed & $\mathrm{Mr}(\exp )$ & $\mathrm{Mr}(\mathrm{cal})$ & Peptide \\
\hline P1 & 445 & 521 & 8179.66 & 8179.66 & 8179.89 & $\begin{array}{l}\text { K.GCNRGVLTMGWGSGTSKLPYLITPQEAIANITPTA } \\
\text { EFFITDSFPSSVDANDEDIAIVFINSDSGENYITVDGNPGDR.K }\end{array}$ \\
P2 & 730 & 777 & 5014.09 & 5014.09 & 5014.35 & $\begin{array}{l}\text { K.TYPYPDGYSTDPQPPPRAGGAEGGNPALWDVAFS } \\
\text { VQVTVTNTGQHSGR.A }\end{array}$ \\
\hline
\end{tabular}


Table 3 Affinity of G2944 (GT-BGL) towards cellobiose and glucose determined through molecular docking studies

\begin{tabular}{llll}
\hline S. no. & Secretory BGL isoform & Affinity to cellobiose $(\mathrm{kcal} / \mathrm{mol})$ & Affinity to glucose $(\mathrm{kcal} / \mathrm{mol})$ \\
\hline 1. & G493 & -6.0 & -5.4 \\
2. & G2944 & -7.5 & -5.7 \\
3. & G3151 & -6.9 & -6.9 \\
4. & G6460 & -7.1 & -5.7 \\
5. & G9078 & -6.8 & -6 \\
6. & G9081 & -6.8 & -5.5 \\
\hline
\end{tabular}

searching in Pfam database. After analysis of the conserved motifs, 17 glycoside hydrolase genes were predicted to be $\beta$-glucosidases. Fourteen of these genes belonged to GH3 family while 3 belonged to the GH1 family. The SignalP program predicted six of these proteins to be secretory (Supplementary Table S1). These predicted secretory BGLs varied in their physicochemical properties (Supplementary Table S2). The molecular weights of the secretory BGLs ranged from 82 to $135 \mathrm{kDa}$. Among the 17 predicted BGLs, initial screening for the glucose tolerant $\beta$-glucosidase was done through peptide similarity search, using the peptide sequences of GTBGL obtained from MALDI-MS data. Translated protein sequence from the GH3 $\beta$-glucosidase gene with gene ID g2944 matched the GT-BGL peptide sequences obtained from MALDI-MS analysis. The peptide P1 from MALDI-MS data showed 100\% query coverage with $91 \%$ sequence identity, while peptide P2 showed $85 \%$ sequence identity with $100 \%$ query coverage to g2944 (Supplementary Fig. S2). No significant matches were obtained with the other BGL sequences; therefore, it was hypothesized that g2944 is the coding sequence for the glucose tolerant BGL3.

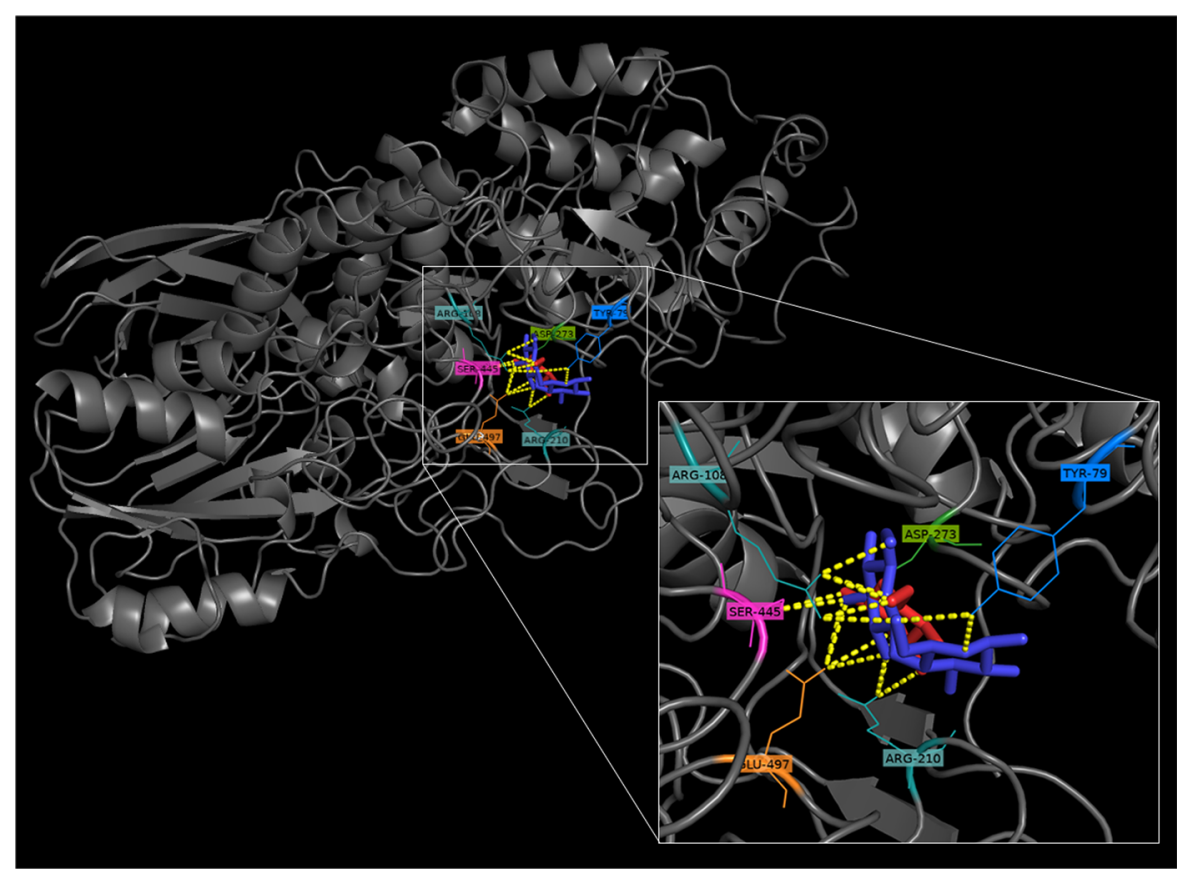

Fig. 4 Molecular Docking Simulation of GT-BGL (G2994) with cellobiose and glucose. The sequence of GT-BGL was modelled using Aspergillus oryzae Family GH3 $\beta$-D-glucosidase (PDB ID: 5FJJ) as the template. Amino acid residues interacting with cellobiose (blue) and glucose (red): Tyr 79 (blue), Arg 108 (Teal), Arg 210 (Teal), Asp 273 (Green), Ser 445 (Pink), and Glu 497 (Orange). Yellow lines show the hydrogen bonds. Groove where ligands bind is shown zoomed in inset for clarity) 

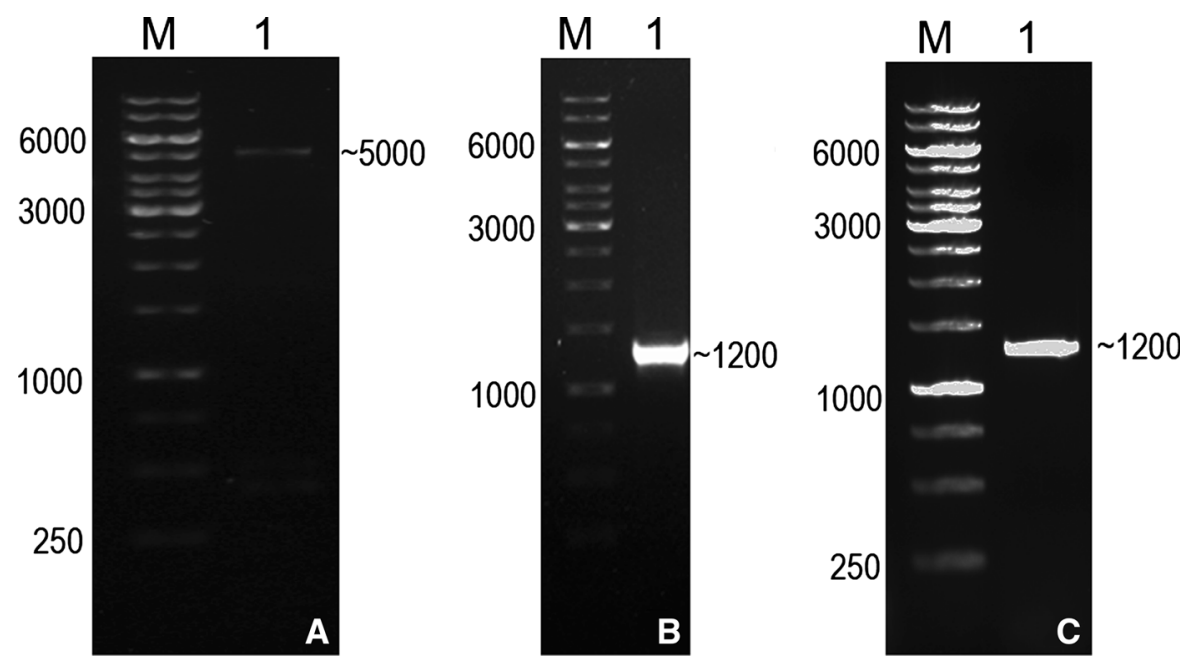

Fig. 5 Confirmation of the presence of GT-BGL (g2944) in the A. unguis genome and its expression by PCR amplification of the gene using genomic and cDNA templates. PCR amplification of full length ( $5000 \mathrm{bp}$ ) gene of GT-BGL (g2944) from A. unguis genomic DNA (a), amplification of catalytic domain of

\section{Docking studies}

3D models of the six secretory BGL proteins of $A$. unguis were generated through homology modeling at the Swiss Modeler Homology Modeling server using appropriate templates (Supplementary Table S3). Insilico docking studies with the substrate (cellobiose) and product (glucose) was performed to determine the affinity towards the substrate and the product, so as to identify the GT-BGL protein and to determine the amino acid residues interacting with the substrate and the product. The binding affinity to cellobiose was highest for the protein G2944 compared to the other secretary BGLs and this protein model showed a comparatively lower affinity for glucose confirming that G2944 could indeed be the GT-BGL (Table 3). As per the predicted model of G2944 (the speculated GTBGL), cellobiose and glucose binds to Tyr 79, Arg 108, Arg 210, Asp 273, Ser 445, and Glu 497 (Fig. 4). All these residues except Tyr 79, Ser 445 and Glu 497 are part of the $N$ terminal glycosyl hydrolase domain of BGL. The latter two amino acids among these belong to the GH3 $C$ terminal (catalytic) domain.
G2944 from A. unguis genomic DNA (b), amplification of catalytic domain of G2944 from cDNA of Xylan grown cultures. $\mathrm{M}=$ O'GeneRuler $1 \mathrm{~kb}$ DNA ladder (Thermo Scientific), Lane 1 = Amplicons from respective experiments

Amplification of GT-BGL from genomic DNA and cDNA

The putative GT-BGL sequence g2944 (BGL3) is a $4309 \mathrm{bp}$ long nucleotide sequence. The BLASTX analysis of the sequence revealed $\sim 85 \%$ identity to $\beta$-1,4-glucosidase from A. nidulans FGSC A4, and other similar proteins. Conserved domains were detected by PROSITE analysis, including a $\beta$-Dglucoside glucohydrolase domain (residues 3-815), the GH3 $N$-terminal domain (residues 92-332) and the GH3 $C$-terminal domain (residues 394-623), which is involved in catalysis and may be involved in binding $\beta$-glucan; and the DUF572 domain which is a eukaryotic protein domain present in proteins required under certain conditions (residues 873-1092).

The complete sequence was amplified from the genomic DNA of $A$. unguis using the primer pair BGLFF1-BGLFR1 which gave an 5000 bp amplicon (Fig. 5a). The catalytic domain (Glycosyl hydrolase family $3 C$-terminal domain) was also amplified using the primer pair BGLCAT_F1-BGLCAT_R1 and this gave an $\sim 1200 \mathrm{bp}$ amplicon as expected (Fig. 5b). Amplification of the catalytic domain was also performed using cDNA of the xylan-induced culture as template using the same primer pair, which gave the $\sim 1200 \mathrm{bp}$ amplicon, which was then 
sequenced and confirmed as the GH3 family $\beta$ glucosidase (Fig. 5c). On alignment using CLUSTALW, it was observed that the peptide P1 is also a part of this domain, thus confirming our prediction that g2944 encodes BGL3 (Supplementary Fig. S3).

\section{Discussion}

The Aspergillus genus is well known for the secretion of different carbohydrate active enzymes (CAZymes) including BGLs. BGLs produced by Aspergilli have been widely studied and are used industrially. The BGL secreted by $A$. niger is considered as a standard in commercial $\beta$-glucosidase production (Sørensen et al. 2013). Since BGLs catalyze the rate-limiting step in the hydrolysis of cellulose and are susceptible to product inhibition, there is a lot of scope for utilizing $\beta$-glucosidases with glucose tolerance to develop highly potent cellulase enzyme cocktails.

There are several reports of other yeast and fungal strains producing glucose tolerant $\beta$-glucosidase (GTBGL), notably the extracellular $\beta$-glucosidase from Candida peltata (NRRL Y-6888), which has a $\mathrm{K}_{\mathrm{i}}$ of $1.4 \mathrm{M}(252 \mathrm{mg} / \mathrm{ml})$ (Saha and Bothast 1996), and the $\beta$-glucosidase from Aspergillus niger CCRC 31494 with a $\mathrm{K}_{\mathrm{i}}$ of $0.543 \mathrm{M}$. The latter enzyme also has the added advantage of not being inhibited by other sugars like fructose, galactose, mannose, and xylose (Yan and Lin, 1997). In comparison, the strain used in this study-A. unguis NII 08123, produces a highly glucose tolerant $\beta$-glucosidase (BGL3) with a glucose inhibition constant $(\mathrm{Ki})$ of $0.8 \mathrm{M}$, which is the second highest tolerance to glucose reported amongst BGLs from the A. nidulans family.

The BGL enzyme preparation from A. unguis also improved the rate of lignocellulose hydrolysis. The rate of glucose release was much higher, while using cellulase cocktails supplemented with A. unguis BGL as compared with commercial BGL supplementation. Another important observation was that on using the commercial enzyme mixture, there was a rapid drop in sugar concentration after $24 \mathrm{~h}$, probably due to the conversion of glucose back to di- or oligosaccharides by the trans-glycosylation activity of BGLs in that preparation. Compared to this, the A. unguis enzyme supported peak yields for longer durations (up to $36 \mathrm{~h}$ ) which is important for biomass conversion to fuels/chemicals, since the hydrolysate normally has to undergo further unit operations before the final product recovery. On the whole, the Aspergillus unguis BGL preparation released approximately 1.6 times more sugar than the commercial BGLs within a $12 \mathrm{~h}$ period.

In addition to deconstruction of biomass, BGLs have other intracellular functions and several isoforms of this enzyme are normally secreted by fungi. The SDS-PAGE analysis of different strains of A. niger showed different BGLs ranging in molecular weight between 68 and $220 \mathrm{kDa}$ (Narasimha et al. 2016), whereas in Penicillium italicum a $354 \mathrm{kDa} \beta$-glucosidase was identified (Park et al. 2012). $\beta$-Glucosidases predicted from the genome of $A$. unguis in this study, range in molecular weight from 82 to $135 \mathrm{kDa}$.

MALDI-TOF analysis of BGL3 indicated that it has a molecular weight of approximately $15 \mathrm{kDa}$. Almost all BGLs deposited so far in SWISS-PROT and NCBI non-redundant databases have a molecular weight above $20 \mathrm{kDa}$. BGL3 therefore may be considered as a rare example of a low molecular weight glucose tolerant $\beta$-glucosidase. Interestingly, comparison of the peptide sequences with the whole genome data showed that they are part of a predicted $135 \mathrm{kDa} \mathrm{GH} 3$ family $\beta$-glucosidase. We therefore speculate that this difference in the molecular weights of observed and predicted proteins may be because of a post translational modification.

The in silico characterization of the protein also confirmed that the protein has the ability to withstand high concentrations of its product glucose. Docking simulations indicated that the glucose tolerant $\beta$ glucosidase has lesser binding affinity for its product glucose $(-5.7 \mathrm{kcal} / \mathrm{mol})$ compared to the affinity for its substrate cellobiose $(-7.5 \mathrm{kcal} / \mathrm{mol})$. There are very few reports available regarding the mechanism of glucose tolerance. A novel $\beta$-glucosidase (Bg16) with an IC-50 of 3.5 M against glucose was identified from a metagenomic library (Cao et al. 2015). Homology models of two GH1 $\beta$-glucosidases Bgl1A and Bgl1B, previously identified from bacteria were used to study the mechanism of glucose tolerance by Fang et al. (2010). The simulated models of Bgl1A and Bgl1B showed differences in eight sequences around the substrate channel. The two proteins were then mutated by interchanging these sequences between them. In Bgl1A there was no direct correlation between glucose tolerance and the eight amino acid residues. Surprisingly, in Bgl1B, mutations in two sequences- B4 
$\left(\mathrm{V}_{227} \mathrm{H}_{228}\right.$ mutated to $\left.\mathrm{F}_{227} \mathrm{~T}_{228}\right)$ and $\mathrm{B} 5\left(\mathrm{~N}_{301} \mathrm{~V}_{302}\right.$ to $\mathrm{Q}_{301} \mathrm{~F}_{302}$ )-led to a surge in glucose tolerance. Further analysis using single-point interchange mutants showed that the change $\mathrm{H}_{228} \mathrm{~T}$ is accountable for the tolerance.

From the study of Bgl1 A and Bgl1B mutants $\mathrm{H}_{228} \mathrm{~T}$ and $\mathrm{N}_{301} \mathrm{Q} / \mathrm{V} 3_{02} \mathrm{~F}$, it was observed that in both cases, glucose binds outside of the active site, most preferably at the middle/entrance of the channel; reducing the chances of competitive inhibition between the substrate and the product probably accounting for the glucose tolerance. Binding of glucose to the middle of the channel can also stimulate substrate cleavage by trans glycosylation (Yang et al. 2015). However, these studies also revealed that glucose tolerance is influenced by other factors like folding because molecular docking of Bgl1A with glucose recommended several possible binding positions with different preferences.

In our docking analysis, it was found that the G2944-cellobiose complex was stabilized by means of hydrogen bonding with Tyr 79, Arg 108, Arg 210, Asp 273, Ser 445, and Glu 497, while all these residues except Tyr 79 were also involved in enzyme-substrate interactions in the G2944-Glucose complex (Fig. 4). All these residues except Tyr 79, Ser 445 and Glu 497 are part of the $N$ terminal glycosyl hydrolase domain of BGL; Ser 445 and Glu 497 belong to the GH3 $C$ terminal (catalytic) domain. Apparently, from the observation of glucose and cellobiose binding to the possible catalytic groove, it seems the amino acid residues of the enzyme interacting with these molecules are similar indicating the reasons for product inhibition. At the same time, the protein has a higher affinity for cellobiose compared to glucose, which could be the reason for its higher glucose tolerance. However, current results are in sufficient to draw conclusions on the molecular reasons for higher glucose tolerance and further efforts are on to elucidate this.

Acknowledgements PKV wishes to thank CSIR for providing research fellowship to pursue his PhD studies at NIIST. We are thankful to Department of Biotechnology, Govt. of India for $\mathrm{R} \& \mathrm{D}$ funding to RKS under the project BT/PR20695/BBE/117/ $211 / 2016$. The $P$. janthinellum strain is a kind gift from Dr. D. V. Gokhale, from the CSIR-National Chemical Laboratory, Pune, India. We thank Dr. N Ramesh Kumar, MPTD, CSIR-NIIST for sequencing services and Mass Spectrometry and Proteomic Core Facility, Rajiv Gandhi Centre for Biotechnology (RGCB), Thiruvananthapuram for the MALDI-TOF analysis.

\section{Compliance with ethical standards}

Ethical approval This study was partially funded by Department of Biotechnology Govt. of India (BT/PR20695/ BBE/117/211/2016). RKS, the corresponding author declare on behalf of all authors that we do not have any conflict of interest. This article does not contain any studies with human participants or animals performed by any of the authors.

\section{References}

Agirre J, Ariza A, Offen WA, Turkenburg JP, Roberts SM, McNicholas S, Harris PV, McBrayer B, Dohnalek J, Cowtan KD, Davies GJ, Wilson KS (2016) Three-dimensional structures of two heavily $\mathrm{N}$-glycosylated sp. family GH3 $\beta$-d-glucosidases. Acta Crystallogr Section D Struct Biol 72(2):254-265

Amore A, Giacobbe S, Faraco V (2013) Regulation of cellulase and hemicellulase gene expression in fungi. Curr Genom 14:230-249. https://doi.org/10.2174/ 1389202911314040002

Bailey TL, Williams N, Misleh C, Li WW (2006) MEME: discovering and analyzing DNA and protein sequence motifs. Nucleic Acids Res 34:W369-W373. https://doi. org/10.1093/nar/gk1198

Biasini M, Bienert S, Waterhouse A, Arnold K, Studer G, Schmidt T, Kiefer F, Cassarino TG, Bertoni M, Bordoli L, Schwede T (2014) SWISS-MODEL: modelling protein tertiary and quaternary structure using evolutionary information. Nucleic Acids Res 42:W252-W258. https://doi. org/10.1093/nar/gku340

Bradford MM (1976) A rapid and sensitive method for the quantitation of microgram quantities of protein utilizing the principle of protein-dye binding. Anal Biochem 72:248-254. https://doi.org/10.1016/00032697(76)90527-3

Cao L-C, Wang Z-J, Ren G-H, Kong W, Li L, Xie W, Liu Y-H (2015) Engineering a novel glucose-tolerant $\beta$-glucosidase as supplementation to enhance the hydrolysis of sugarcane bagasse at high glucose concentration. Biotechnol Biofuels 8:202. https://doi.org/10.1186/s13068-015-0383-z

Christopher M, Mathew AK, Kiran Kumar M, Pandey A, Sukumaran RK (2017) A biorefinery-based approach for the production of ethanol from enzymatically hydrolysed cotton stalks. Bioresour Technol 242:178-183. https://doi. org/10.1016/j.biortech.2017.03.190

DeLano WL (2002) The PyMOL molecular graphics system. DeLano Scientific LLC, San Carlos

Fang Z, Fang W, Liu J, Hong Y, Peng H, Zhang X, Sun B, Xiao Y (2010) Cloning and characterization of a $\beta$-glucosidase from marine microbial metagenome with excellent glucose tolerance. J Microbiol Biotechnol 20:1351-1358. https:// doi.org/10.4014/jmb.1003.03011

Gasteiger E, Gattiker A, Hoogland C, Ivanyi I, Appel RD, Bairoch A (2003) ExPASy: the proteomics server for indepth protein knowledge and analysis. Nucleic Acids Res 31:3784-3788 
Ghose TK (1987) Measurement of cellulase activities. Pure Appl Chem 59:257-268. https://doi.org/10.1351/ pac198759020257

Goodsell DS, Olson AJ (1990) Automated docking of substrates to proteins by simulated annealing. Proteins Struct Funct Genet 8:195-202. https://doi.org/10.1002/prot.340080302

Guex N, Peitsch MC (1997) SWISS-MODEL and the Swiss-Pdb viewer: an environment for comparative protein modeling. Electrophoresis 18:2714-2723. https://doi.org/10.1002/ elps. 1150181505

Mandels M, Weber J (1969) The production of cellulases. Adv Chem Ser 95:391-414

Miller G (1959) Use of dinitrosalisylic acid reagent for determination of reducing sugar. Anal Chem 31:426-428

Narasimha G, Sridevi A, Ramanjaneyulu G, Rajasekhar Reddy B (2016) Purification and characterization of $\beta$-glucosidase from Aspergillus niger. Int J Food Prop 19:652-661. https://doi.org/10.1080/10942912.2015.1023398

Park A-R, Hong JH, Kim J-J, Yoon J-J (2012) Biochemical characterization of an extracellular $\beta$-glucosidase from the fungus, Penicillium italicum, isolated from rotten citrus peel. Mycobiology 40:173-180. https://doi.org/10.5941/ MYCO.2012.40.3.173

Petersen TN, Brunak S, von Heijne G, Nielsen H (2011) SignalP 4.0: discriminating signal peptides from transmembrane regions. Nat Methods 8:785-786. https://doi.org/10.1038/ nmeth. 1701

Rajasree KP, Mathew GM, Pandey A, Sukumaran RK (2013) Highly glucose tolerant $\beta$-glucosidase from Aspergillus unguis: NII 08123 for enhanced hydrolysis of biomass. J Ind Microbiol Biotechnol 40:967-975. https://doi.org/10. 1007/s10295-013-1291-5

Saha BC, Bothast RJ (1996) Production, purification, and characterization of a highly glucose-tolerant novel $\beta$-glucosidase from Candida peltata. Appl Environ Microbiol 62:3165-3170
Saloheimo M, Kuja-Panula J, Ylosmaki E, Ward M, Penttila M (2002) Enzymatic properties and intracellular localization of the novel Trichoderma reesei $\beta$-glucosidase BGLII (Cel1A). Appl Environ Microbiol 68(9):4546-4553

Singh A, Kumar Patel A, Adsul M, Rani Singhania R (2017) Genetic modification: a tool for enhancing cellulase secretion. Biofuel Res J 14:600-610. https://doi.org/10. 18331/BRJ2017.4.2.5

Sørensen A, Lübeck M, Lübeck P, Ahring B (2013) Fungal betaglucosidases: a bottleneck in industrial use of lignocellulosic materials. Biomolecules 3(4):612-631

Tabanag ID, Tsai S-L (2018) Hemicellulose by a synthetic saccharomyces cerevisiae consortium. bioRxiv. https://doi. org/10.1101/244244

Tiwari P, Misra BN, Sangwan NS (2013) $\beta$-glucosidases from the fungus Trichoderma: an efficient cellulase machinery in biotechnological applications. Biomed Res Int. https:// doi.org/10.1155/2013/203735

Trauger SA, Webb W, Siuzdak G (2002) Peptide and protein analysis with mass spectrometry. Spectroscopy 16:15-28

Wu L, Moteki T, Gokhale AA, Flaherty DW, Toste FD (2016) Production of fuels and chemicals from biomass: condensation reactions and beyond. Chem 1:32-58. https://doi. org/10.1016/J.CHEMPR.2016.05.002

Yan TR, Lin CL (1997) Purification and characterization of a glucose-tolerant $\beta$-glucosidase from Aspergillus niger CCRC 31494. Biosci Biotechnol Biochem 61:965-970

Yang Y, Zhang X, Yin Q, Fang W, Fang Z, Wang X, Zhang X, Xiao Y (2015) A mechanism of glucose tolerance and stimulation of GH1 $\beta$-glucosidases. Sci Rep. https://doi. org/10.1038/srep17296

Publisher's Note Springer Nature remains neutral with regard to jurisdictional claims in published maps and institutional affiliations. 